\title{
Temperature and Size Effects on Diffusion in Carbon Nanotubes
}

\author{
S. Jakobtorweihen, ${ }^{*}$, F. J. Keil, ${ }^{\dagger}$ and B. Smit ${ }^{\ddagger}, \S$ \\ Chemical Reaction Engineering, Hamburg University of Technology, Eissendorfer Str. 38, D-21073 Hamburg, \\ Germany, Van't Hoff Institute for Molecular Sciences (HIMS), University of Amsterdam, Nieuwe Achtergracht \\ 166, 1018 WV Amsterdam, The Netherlands, and ${ }^{3}$ CECAM (Centre Européen de Calcul Atomique Moléculaire), \\ Ecole Normale Supérieure, 46 Allée d'Italie, 69364 Lyon Cedex 7, France
}

Received: June 2, 2006; In Final Form: July 5, 2006

\begin{abstract}
We study the self-diffusion of simple gases inside single-walled carbon nanotubes at the zero-loading limit by molecular dynamics simulations. The host-framework flexibility influence is taken into account. In particular, we study the influences of nanotube size and temperature. For the carbon-nanotube radius-dependent selfdiffusivities, a maximum is observed, which resembles the so-called levitation effect. This occurs for pores having a radius comparable to the position of the interaction-energy minimum. Surprisingly, the temperature influence is not uniform throughout different pore sizes. Diffusivities are expected to increase with temperature. This effect is observed for carbon nanotubes distinctly larger than the guest molecules. Remarkably, for smaller pores, the self-diffusivities decrease with increasing temperature or exhibit a maximum in the temperature dependence. This effect is caused by competing influences of collision frequency and temperature.
\end{abstract}

\section{Introduction}

Carbon nanotubes (CNTs) can be aligned to form a wellordered nanoporous membrane $\mathrm{e}^{1,2}$ that can be incorporated in a macroscopic structure ${ }^{3}$ for separation devices. Because of these applications, the behavior of guest molecules inside carbon nanotubes has recently attracted considerable attention. ${ }^{4-11}$ Since the diffusion shows a strong dependence on the ratio of the CNT diameter and the sorbate size, a dynamical separation of the different sorbates would be possible. The behavior of fluids inside these and other nanoporous materials is quite different from the bulk phase, and our understanding of motion inside nanoscale pores is still fragmentary. In this work we show some remarkable phenomena in the diffusive behavior of guest molecules in nanoscale materials; e.g., for some CNTs an increased temperature will lead to reduced self-diffusivities.

In this article, we focus on the zero-loading limit and examine the influence of pore radius and temperature on self-diffusion of simple molecules. At low loadings (high Knudsen numbers), the diffusion process is completely controlled by fluid-pore collisions. ${ }^{10}$ Interestingly, even at such low loadings, the diffusion behavior exhibits very complex phenomena.

Derouane and co-workers ${ }^{12,13}$ were the first to mention an enhanced mobility for cases where the dimensions of guest particles closely match the host structure dimensions. They called this effect the "floating molecule". Yashonath and coworkers examined this effect in more detail for diffusion in zeolites ${ }^{14-17}$ using molecular dynamics simulations. They termed this behavior the "levitation effect". If the guest molecule fits perfectly in the window of the zeolite, the molecule appears to be "floating"; increasing or decreasing the diameter of the guest molecules causes the diffusion coefficient to decrease. It was shown that this effect is temperature dependent. ${ }^{17} \mathrm{~A}$ similar

\footnotetext{
* Corresponding author, e-mail: jakobtorweihen@tuhh.de.

† Hamburg University of Technology.

$\div$ University of Amsterdam.

$\S$ CECAM.
}

effect has been observed by Bhide et al. ${ }^{18}$ for carbon nanotubes. In this simulation study, a crossover from diffusive behavior to superdiffusive behavior for an increasing sorbate size was shown.

Simulations of diffusion of molecules in nanotubes in the low-density limit are surprisingly difficult. The onset of diffusive behavior occurs at time scales that are much larger compared to other materials. In addition, the movement of the atoms of the CNT has a large influence on the diffusion. ${ }^{10}$ As most of the simulations assume rigid carbon nanotubes, it is important to investigate whether in a more realistic CNT levitation effects occur. First, up to now, levitation in CNTs has been investigated only in rigid CNTs, by simulating the nanotube as flexible framework, the results are distinctly different. Second, for industrial applications it is important to know under which conditions diffusion is enhanced or lowered. Third, zeolites have wide regions (cages) and narrow regions (windows). CNTs have a constant radius. In particular, we show that the temperature influence on the self-diffusivities is different in CNTs than in zeolites.

We study phenomena of guest molecules inside CNTs in detail by means of molecular dynamics simulations. The investigated systems show normal-mode diffusion and confirm that a levitation effect can be observed in the self-diffusivities. In contrast, Bhide et al. ${ }^{18}$ have observed that the diffusion mode changes with guest size and stated that this change is the levitation effect. In addition, we found that the temperature influence is different for various CNTs. In some cases, selfdiffusion is increased with temperature. Surprisingly, in some cases, $D_{\mathrm{S}}$ is decreasing with increasing temperature, and in other cases, a small maximum can be observed in the temperature dependence.

The outline of this article is as follows: In the next section, the models underlying our simulations are explained, and details of the simulations are given. In section 3 , the results are shown and discussed. We end with a summary in the last section. 


\section{Methods}

Very recently we have shown that at low loadings the CNT "flexibility" is always relevant. It has a crucial influence on the diffusive dynamics of the guest molecules. ${ }^{10}$ Additionally, we have introduced a very efficient algorithm to avoid the expensive flexible CNT simulations. ${ }^{10,11}$ This method results in, effectively, the same diffusivities and other effects as obtained from flexible CNT simulations. It uses a LoweAndersen thermostat ${ }^{19}$ which works on interface-fluid collisions. The central idea of this "Lowe-Andersen interface-fluid collision (LA-IFC) thermostat" is to mimic the thermal effects of a flexible framework stochastically. Chen et al. ${ }^{8}$ have used this approach to show the influence of CNT flexibility on transport diffusion.

Since we are concentrating on the zero-loading limit, it is important to take the flexibility effect into account. We use the LA-IFC thermostat as described in ref 11. The thermostat parameters used for this work are shown in the appendix. The simulations were performed with 64 ideal, i.e., noninteracting, fluid particles. We carefully checked that the results are independent of the particle number. All self-diffusion coefficients, $D_{\mathrm{S}}$, are calculated only for motion parallel to the pore axis by means of the Einstein equation. ${ }^{20}$ Further details of the simulations are described in ref 11 . To show that our results reproduce the flexibility influence correctly and to demonstrate that the observed effects are not artifacts of the thermostat, some systems were simulated with a flexible CNT. The size and helicity of carbon nanotubes is specified by two integer numbers $n_{1}$ and $n_{2},{ }^{21}$ so they can be unambiguously labeled as $\left(n_{1}, n_{2}\right)$. All results in this work were obtained with single walled carbon nanotubes in a zigzag structure.

To model a carbon nanotube as flexible framework, we followed Walther et al., ${ }^{22}$ employing a small modification which is described in ref 11. Intermolecular interactions were modeled with the shifted and truncated Lennard-Jones (LJ) 12-6 potential, for which a cutoff radius of $14 \AA$ was used. The fluid molecules were modeled as united-atoms. The LJ parameters for methane $\left(\sigma_{\mathrm{CH}_{4}}=3.73 \AA\right.$, $\left.\epsilon_{\mathrm{CH}_{4}} / k_{\mathrm{B}}=148 \mathrm{~K}\right)$ were taken from ref 23. The LJ parameters for helium $\left(\sigma_{\mathrm{He}}=2.551 \AA, \epsilon_{\mathrm{He}} / k_{\mathrm{B}}=\right.$ $10.22 \mathrm{~K}$ ) were taken from ref 24 . The carbon LJ parameters $\left(\sigma_{\mathrm{C}}=3.4 \AA, \epsilon_{\mathrm{C}} / k_{\mathrm{B}}=28 \mathrm{~K}\right)$ were taken from ref 25 . The Lorentz-Berthelot mixing rules were used to determine the interactions of unlike molecular centers.

It is very simple to relate our formalism of the LA-IFC thermostat ${ }^{11}$ to the Stokes-Einstein-equation (SEE). By using $\gamma=\Gamma \mu,{ }^{11}$ the SEE can be rewritten as

$$
D_{\mathrm{S}}=\frac{T k_{\mathrm{B}}}{\Gamma \mu}
$$

where $D_{\mathrm{S}}$ is the self-diffusion coefficient, $T$ is the temperature, $k_{\mathrm{B}}$ the Boltzmann constant, $\gamma$ the friction coefficient, $\Gamma$ the collision frequency, and $\mu=m_{\mathrm{f}} m_{\mathrm{C}} /\left(m_{\mathrm{f}}+m_{\mathrm{C}}\right)$ is the reduced mass, with $m$ the mass of a particle, $\mathrm{f}$ indicates a fluid molecule, and $\mathrm{C}$ a carbon atom. By using the collision frequency, $\Gamma_{z, f l e x}$, obtained from short flexible CNT simulations ${ }^{11}$ the results from "full" simulations can be reproduced by the SEE (see Figures 2 and 5). Note, that $\Gamma_{z, f l e x}$ is not the same as the input parameters for the LA-IFC thermostat (for more details see ref 11). To distinguish between the different simulation methods we adopt the following conventions: Simulations including a fully flexible carbon nanotube are denoted as flexible CNT simulations, simulations with a rigid CNT that take the flexibility into account via the interface-fluid collision thermostat are termed
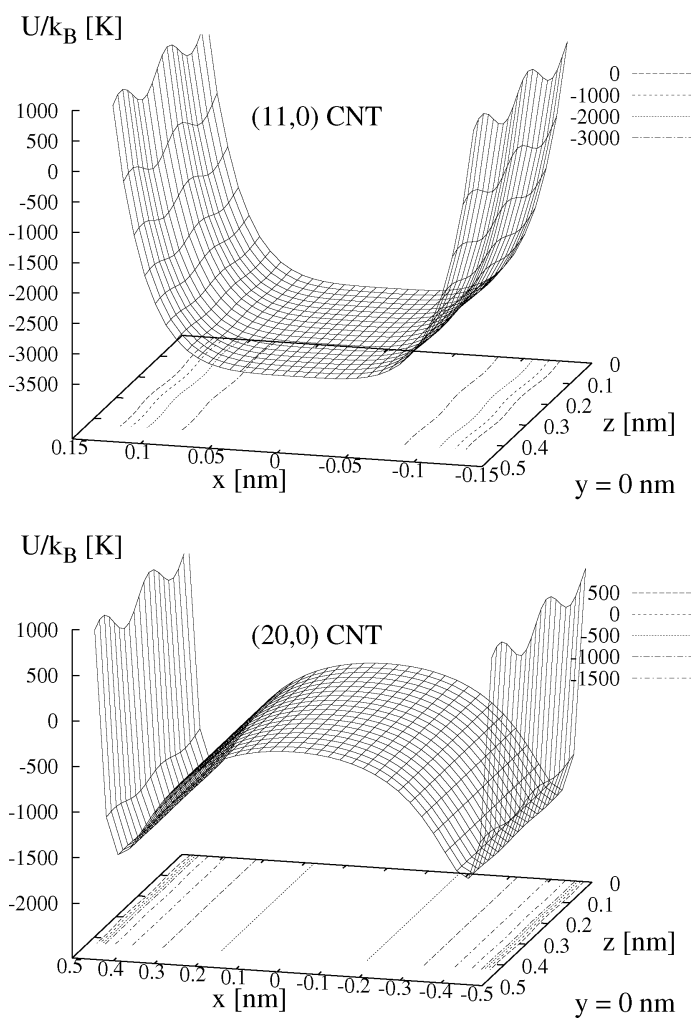

Figure 1. Potential energy of methane inside a $(11,0) \mathrm{CNT}$ (top) and a $(20,0)$ CNT (bottom). The $z$ direction is the direction parallel to the pore axis; the $y$ coordinate is set to zero. The potential energy at each position is caused by the interaction of one methane molecule with the carbon atoms of the nanotube.

"LA-IFC" simulations. Results calculated via the StokesEinstein-equation are labeled as "SEE".

\section{Results and Discussion}

In Figure 1 the molecular potential fields for methane inside two different CNTs are shown. For narrow CNTs the potential minimum is located at the center of the tube. For broader CNTs the minimum is located near the pore walls. Therefore, molecules adsorb at the center in small tubes rather than at the pore wall. The minimum is flatter for the $(11,0)$ CNT than for $(20,0) \mathrm{CNT}$. For tubes smaller than the $(11,0) \mathrm{CNT}$ the potential profile is less flat.

In Figure 2, the dependence of methane self-diffusion on the $\mathrm{CNT}$ radius, $r_{\mathrm{CNT}}$, is presented for the zero-loading limit and for different temperatures. The diffusivities are decreasing with decreasing $r_{\mathrm{CNT}}$ for the larger radii, as predicted by the Knudsen model. By further decreasing the radius, $D_{\mathrm{S}}$ starts to increase until a maximum value is reached. Thereafter, it declines again. At $300 \mathrm{~K}$ for distances between 19.6 and $23.5 \AA$ only a very small increase of $D_{\mathrm{S}}$ is observed, here the diffusivities just start to increase. For larger radii, the increase is more pronounced (not shown). For $700 \mathrm{~K}$ the local maximum (levitation) is not as pronounced as for the other temperatures investigated. It is very likely that the maximum will completely vanish for higher temperatures. The position of the maximum corresponds to a radius which is slightly larger than the position of the LennardJones potential minimum $2^{1 / 6} \sigma_{\mathrm{CH}_{4}-\mathrm{C}}$. Yashonath and co-workers observed a maximum in the size-dependent mobility for diffusion inside zeolites. ${ }^{14-17}$ They termed this behavior the levitation effect and introduced a so-called levitation parameter $\gamma=2^{1 / 6} \sigma_{\mathrm{gh}} / r_{\mathrm{CNT}}$, where $\sigma_{\mathrm{gh}}$ is the Lennard-Jones size parameter for the guest-host interaction. They showed that the maximum 


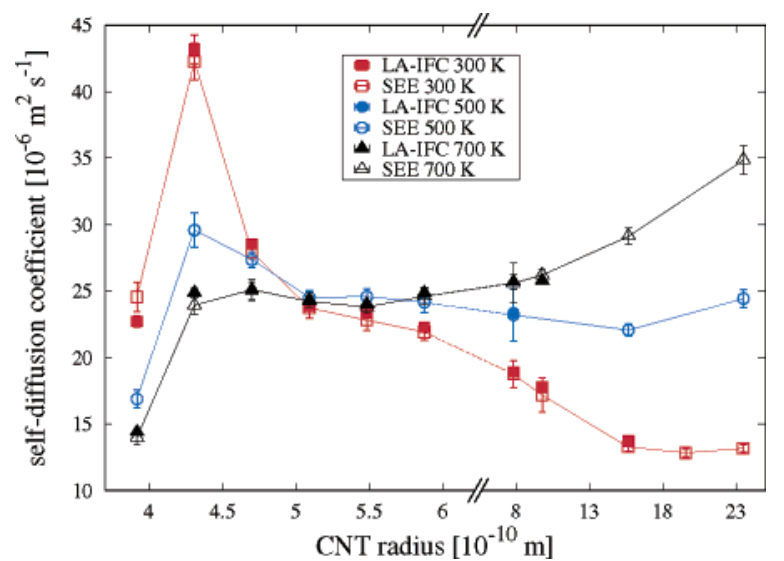

Figure 2. Self-diffusion coefficients of $\mathrm{CH}_{4}$ at zero loading for three temperatures. Results from simulations where the flexibility influence is taken into account via the LA-IFC thermostat and results obtained with the Stokes-Einstein equation (SEE) are compared. Note that the abscissa is not uniform over the whole range. Lines are added to guide the eye.
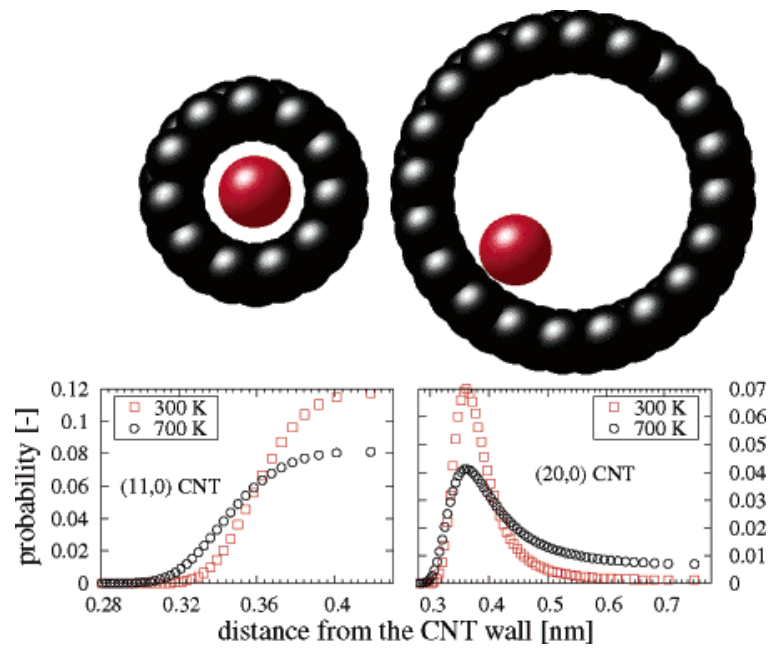

Figure 3. Top: Snapshots of a methane molecule at the most probable position inside a $(11,0) \mathrm{CNT}$ (left) and a $(20,0) \mathrm{CNT}$ (right), shown in the $x y$-plane. The $(11,0) \mathrm{CNT}$ has a radius of $0.43 \mathrm{~nm}$ and the $(20,0)$ of $0.78 \mathrm{~nm}$. For the latter the particle-CNT distance is $0.36 \mathrm{~nm}$ and the molecule inside the $(11,0) \mathrm{CNT}$ is located at the center. Bottom: Probability of finding a particle inside a $(11,0)$ CNT (left) and inside a $(20,0)$ CNT (right). The probabilities were calculated from radial density distributions, which were sampled by a molecular dynamics simulation at the zero-loading limit. Note that the absolute probabilities are dependent on the sectioning; here the pores are divided into hollow cylinders of equal volume and the same number of cylinders is used for the same pore type. The sum of the probabilities is one for each series.

in diffusion occurs for levitation parameters near unity. The value of the levitation parameter at the maximum for the $\mathrm{CH}_{4}-$ CNT system is 0.93 .

To understand this effect we have to consider the positions of the molecules inside the CNTs in detail, in particular the distance to the pore wall. The $(11,0) \mathrm{CNT}$ is the tube for which the maximum is observed. Corresponding to the results of the radial density distribution, the most probable position of the (noninteracting) particles in the plane perpendicular to the axis is located at the center of the pore, see Figure 3 . Hence, the most probable pore-fluid distance $(0.43 \mathrm{~nm})$ is equal to $r_{\mathrm{CNT}}$. For larger CNT radii the most probable position is not the pore center. By enlarging the pore diameter the distance of methane to the wall decreases and becomes constant at approximately

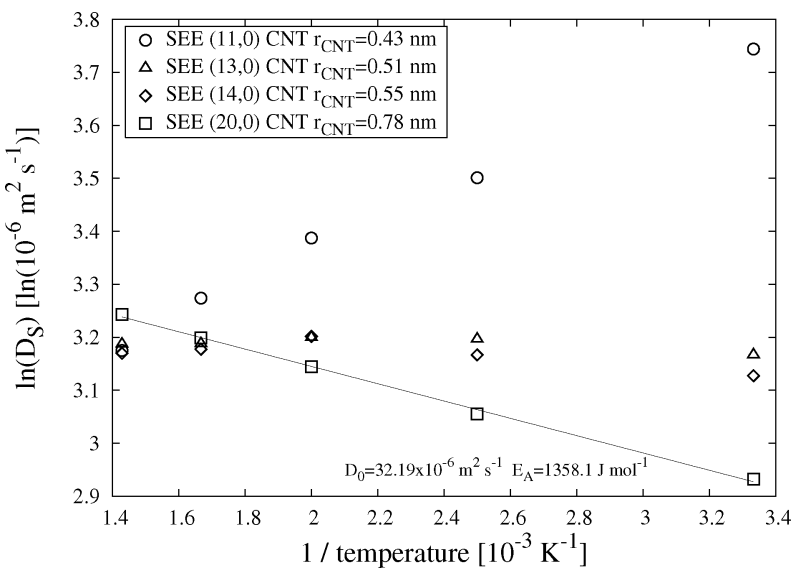

Figure 4. Arrhenius plot for the self-diffusivities of $\mathrm{CH}_{4}$ at zero loading for four CNTs. All results are obtained from the Stokes-Einstein equation (SEE). The values for the activation energy $\left(E_{\mathrm{A}}\right)$ and the preexponential factor $\left(D_{0}\right)$, obtained from a linear fit to the results of the $(20,0) \mathrm{CNT}$, are shown.

$0.36 \mathrm{~nm}$ (see Figure 3). Therefore, a fluid particle collides less frequently with the CNT in the $(11,0)$ CNT than in larger CNTs (lower collision frequency, lower friction). As the motion inside a $(11,0)$ CNT is less perturbed by wall collisions, this leads to a faster diffusion. Another factor is the increasing smoothness with decreasing pore radius, though the influence of this aspect is not as large as the difference in wall distances. The $(10,0)$ CNT is even smoother than the $(11,0)$ CNT, but the selfdiffusion coefficient is lower. For the former the most probable distance from the pore wall is equal to the tube radius. It is larger than $0.36 \mathrm{~nm}$, but smaller than in the case of a $(11,0)$ CNT. Furthermore, this pore is so narrow that the fluid particle "feels" the corrugation of the wall at every position. Hence, the diffusivities are lower in the $(10,0)$ pore compared to the $(11,0)$. The intensity of the maximum in the self-diffusion coefficient depends strongly on temperature. The higher the temperature, the less pronounced the peak. Yashonath and Rajappa ${ }^{17}$ reported the same trend for diffusion in a cage-type zeolite.

Surprisingly, the temperature dependence is not uniform throughout the investigated CNTs. Inside tubes having a radius greater than $0.56 \mathrm{~nm}$, the diffusivities are increasing with temperature, as expected. Notably, for the smallest CNTs, the diffusivities are decreasing with increasing temperature, and for others, there is only a slight temperature influence with a small maximum in the temperature dependence. In Figure 4, the Arrhenius plots of four selected CNTs are presented. For the $(20,0)$ CNT the Arrhenius law is fulfilled. In contrast, the selfdiffusivities calculated for the $(11,0)$ CNT are increasing with decreasing temperature. Furthermore, the diffusivities for the $(13,0)$ and $(14,0)$ CNTs show a very weak $T$ dependence; they exhibit a maximum at $500 \mathrm{~K}$.

To understand these effects we first have to realize that the number of collisions per unit of time is always increasing with temperature. Further, considering eq 1, it is obvious that an increment in $T$ will have a direct positive (raising) effect on $D_{\mathrm{S}}$, but also an indirect lowering effect via the collision frequency in the denominator. Hence, an increasing temperature has a lowering and a raising effect on the self-diffusivities. To answer the question whether the self-diffusivities are increasing or decreasing with temperature we have to find out which temperature influence is stronger, the direct influence on $D_{\mathrm{S}}$ or the influence on the collision frequency. If the collision frequency, $\Gamma_{z, f l e x}$, is a linear function of temperature, the self- 


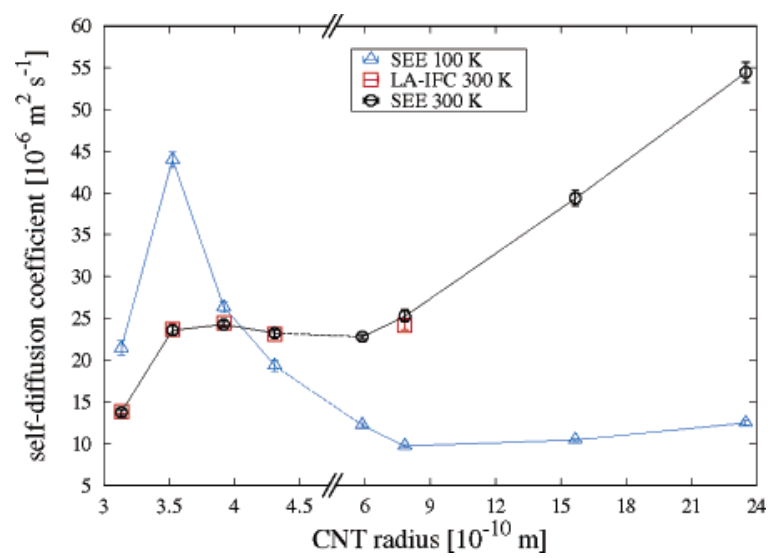

Figure 5. Self-diffusion coefficients of helium at zero loading for two temperatures. Results from simulations where the flexibility influence is taken into account via the LA-IFC thermostat, and results obtained from the Stokes-Einstein equation (SEE) are compared. Note that the abscissa is not uniform over the whole range. Lines are added to guide the eye.

diffusivities are constant over temperature (see eq 1). In cases where this influence is stronger, $D_{\mathrm{S}}$ decreases, and in cases where it is weaker, $D_{\mathrm{S}}$ increases with $T$. On the basis of the results for the $(11,0)$ and $(20,0) \mathrm{CNTs}$, we will explain the different $T$ influences exemplarily. Because of the distribution of the noninteracting particles inside the pores, the effect of an increasing collision frequency with temperature is strengthened in the $(11,0)$ case and weakened in the $(20,0)$ case. The most probable position in the $(20,0)$ CNT (see Figure 3 ) is situated close to the pore wall (at a distance of $0.36 \mathrm{~nm}$ ). Hence, an increase in $T$ will lead to a lower probability of finding the particle close to the pore wall and to a higher probability of finding the particle in the central region (see Figure 3). The situation is different for the $(11,0)$ CNT. As the most probable position inside this tube is located at the pore center, an increase in temperature will result in enhanced probabilities of finding the particle close to the pore wall (see Figure 3 ). Consequently, the increase of the collision frequency due to a faster motion is strengthened by the change of the particle distribution over the pore radius. To highlight this phenomenon quantitatively, we calculate the probability to detect a particle at a fluid-wall distance smaller than $0.35 \mathrm{~nm}$. For methane inside a $(20,0) \mathrm{CNT}$ at $300 \mathrm{~K}$ we find a probability of 0.26 which is diminishing to 0.24 for $700 \mathrm{~K}$. In the $(11,0)$ case this probability is increasing from 0.19 to 0.39 in the same temperature range.

Yashonath and Rajappa ${ }^{17}$ investigated the temperature influence on guest size dependent diffusion in a cage-type zeolite. They showed that the self-diffusivities are always increasing with temperature. But the cage-to-cage crossing rate showed a similar temperature effect as observed for the CNT systems. The cage-to-cage crossing rate comprises only the number of window crossings. It does not take into account the motion inside the cages and the diffusion toward the windows. As the levitation effect is explained for these zeolite systems in terms of window-size/guest-size ratio and for CNT systems in terms of CNT-size/guest-size ratio, it is consequential that the temperature effects in CNTs can be compared to temperature effects on events in the window region. In ref 26 , a minimum in the Arrhenius plot for the cage-to-cage crossing rate was found. This observation was explained by different distances to the zeolite surface.

We observe the same phenomena for helium at the zeroloading limit (see Figure 5) as observed for methane. At 300 $\mathrm{K}$, the intensity of the maximum in the CNT size dependence is not as pronounced as for methane at this temperature. Furthermore, the maximum shifts to smaller CNT radii due to the smaller size of helium.

\section{Summary}

In this work, we have investigated the dynamics of methane and helium at the zero-loading limit inside carbon nanotubes. No fluid-fluid interactions take place at these conditions, so the process is completely controlled by fluid-interface interactions. We have shown that the self-diffusivities exhibit a maximum while plotted against CNT size and that the temperature influence is not unique. For some pores, the self-diffusion coefficients are increasing with temperature, for others, they are decreasing with increasing temperature, and for some, a maximum in the temperature dependence could be observed. These effects have been explained in detail. The decrease of diffusivities with an increased temperature is owing to a stronger temperature influence on collision frequency than on $D_{\mathrm{S}}$ directly (see eq 1). In the case of narrow CNTs, the increase in temperature not only leads to higher collision frequencies due to faster motion, additionally the probability to find a particle close to the pore wall is enhanced (higher collision probability). All self-diffusivities shown in this work were calculated for the direction parallel to the pore axis (one-dimensional). By calculating the diffusivities for the three-dimensional motion, we observe the same effects. Note that for higher loadings in the small CNTs, single-file diffusion will be observed. Therefore, to predict whether the maximum in the size dependence (levitation effect) can be used to optimize technical applications, we have to investigate at which conditions the diffusion mode starts to deviate from the normal mode.

Acknowledgment. We thank E. Beerdsen for her comments on the manuscript. This work was supported by the Deutsche Forschungsgemeinschaft (DFG) in priority program SPP 1155 and by the EC through the Marie Curie EXT project MEXTCT-2005-023311.

\section{Appendix}

a. Thermostat Parameters. We obtained the LA-IFC parameters as described in ref 11. All parameters used for this work are shown in Table 1.

TABLE 1: Parameter Sets for the LA-IFC Thermostat

\begin{tabular}{ccccccc}
\hline component & $\begin{array}{c}T \\
{[\mathrm{~K}]}\end{array}$ & $\mathrm{CNT}$ & $\begin{array}{c}r_{\mathrm{CNT}} \\
{[\mathrm{nm}]}\end{array}$ & $\begin{array}{c}\mathrm{r}_{\mathrm{LA}} \\
{[\mathrm{nm}]}\end{array}$ & $\begin{array}{c}\Gamma_{x y} \\
{\left[10^{11} s^{-1}\right]}\end{array}$ & $\begin{array}{c}\Gamma_{z} \\
{\left[10^{10} s^{-1}\right]}\end{array}$ \\
\hline $\mathrm{CH}_{4}$ & 300 & $(10,0)$ & 0.39 & 0.39 & 0.6 & 1.5 \\
$\mathrm{CH}_{4}$ & 300 & $(11,0)$ & 0.43 & 0.43 & 1.0 & 0.8 \\
$\mathrm{CH}_{4}$ & 300 & $(12,0)$ & 0.47 & 0.38 & 4.5 & 2.3 \\
$\mathrm{CH}_{4}$ & 300 & $(13,0)$ & 0.51 & 0.38 & 4.2 & 3.0 \\
$\mathrm{CH}_{4}$ & 300 & $(14,0)$ & 0.55 & 0.37 & 4.9 & 3.3 \\
$\mathrm{CH}_{4}$ & 300 & $(15,0)$ & 0.59 & 0.37 & 3.8 & 4.0 \\
$\mathrm{CH}_{4}$ & 300 & $(20,0)$ & 0.78 & 0.36 & 3.5 & 5.0 \\
$\mathrm{CH}_{4}$ & 300 & $(25,0)$ & 0.98 & 0.36 & 4.1 & 5.7 \\
$\mathrm{CH}_{4}$ & 300 & $(40,0)$ & 1.57 & 0.36 & 3.3 & 7.3 \\
$\mathrm{CH}_{4}$ & 500 & $(20,0)$ & 0.78 & 0.36 & 5.0 & 7.5 \\
$\mathrm{CH}_{4}$ & 700 & $(10,0)$ & 0.39 & 0.39 & 1.8 & 5.7 \\
$\mathrm{CH}_{4}$ & 700 & $(11,0)$ & 0.43 & 0.43 & 1.8 & 3.3 \\
$\mathrm{CH}_{4}$ & 700 & $(12,0)$ & 0.47 & 0.38 & 6.3 & 5.6 \\
$\mathrm{CH}_{4}$ & 700 & $(13,0)$ & 0.51 & 0.38 & 6.4 & 6.9 \\
$\mathrm{CH}_{4}$ & 700 & $(14,0)$ & 0.55 & 0.37 & 7.2 & 8.0 \\
$\mathrm{CH}_{4}$ & 700 & $(15,0)$ & 0.59 & 0.37 & 5.4 & 9.0 \\
$\mathrm{CH}_{4}$ & 700 & $(20,0)$ & 0.78 & 0.36 & 6.1 & 10.4 \\
$\mathrm{CH}_{4}$ & 700 & $(25,0)$ & 0.98 & 0.36 & 4.3 & 10.4 \\
$\mathrm{He}$ & 300 & $(8,0)$ & 0.31 & 0.31 & 1.9 & 5.4 \\
$\mathrm{He}$ & 300 & $(9,0)$ & 0.35 & 0.35 & 1.3 & 3.1 \\
$\mathrm{He}$ & 300 & $(10,0)$ & 0.39 & 0.32 & 3.2 & 4.3 \\
$\mathrm{He}$ & 300 & $(11,0)$ & 0.43 & 0.31 & 4.9 & 5.5 \\
$\mathrm{He}$ & 300 & $(20,0)$ & 0.78 & 0.30 & 6.5 & 3.8
\end{tabular}


TABLE 2: Comparison of Self-diffusion Coefficients at Zero Loading from Simulations with a Flexible CNT and from Simulations Where the Flexibility Influence Is Taken into Account via the Lowe-Andersen Interface-fluid Collision (LA-IFC) Thermostat ${ }^{a}$

\begin{tabular}{cccccc}
\hline component & $\begin{array}{c}\mathrm{T} \\
{[\mathrm{K}]}\end{array}$ & $\mathrm{CNT}$ & $\begin{array}{c}r_{\mathrm{CNT}} \\
{[\mathrm{nm}]}\end{array}$ & $\begin{array}{c}D_{\mathrm{S}}^{\text {flexible }} \\
{\left[10^{-6} \mathrm{~m}^{2} \mathrm{~s}^{-1}\right]}\end{array}$ & $\begin{array}{c}D_{\mathrm{S}}^{\mathrm{LA}-\mathrm{IFC}} \\
{\left[10^{-6} \mathrm{~m}^{2} \mathrm{~s}^{-1}\right]}\end{array}$ \\
\hline $\mathrm{CH}_{4}$ & 300 & $(10,0)$ & 0.39 & $25.34_{0.95}$ & $22.72_{0.45}$ \\
$\mathrm{CH}_{4}$ & 300 & $(11,0)$ & 0.43 & $43.53_{1.31}$ & $43.18_{1.06}$ \\
$\mathrm{CH}_{4}$ & 300 & $(20,0)$ & 0.78 & $19.42_{0.42}$ & $18.92_{0.31}$ \\
$\mathrm{CH}_{4}$ & 700 & $(11,0)$ & 0.43 & $25.23_{1.05}$ & $24.87_{0.35}$ \\
$\mathrm{He}$ & 300 & $(20,0)$ & 0.78 & $26.18_{1.31}$ & $24.17_{0.57}$
\end{tabular}

${ }^{a}$ Errors are given in in the subscripts.

b. LA-IFC Thermostat Results Compared to Flexible CNT Results. To show that our results reproduce the flexibility influence correctly, and to demonstrate that the observed effects are not artifacts of the thermostat, some systems were simulated with a flexible CNT, see Table 2. As already shown in refs 10 and 11, the simulations using the LA-IFC thermostat result in effectively the same diffusivities.

\section{References and Notes}

(1) Hinds, B. J.; Chopra, N.; Rantell, T.; Andrews, R.; Gavalas, V.; Bachas, L. G. Science 2004, 303, 62-65.

(2) Casavant, M. J.; Walters, D. A.; Schmidt, J. J.; Smalley, R. E. J. Appl. Phys. 2003, 93, 2153-2156.

(3) Srivastava, A.; Srivastava, O. N.; Talapatra, S.; Vajtai, R.; Ajayan, P. Nat. Mater. 2004, 3, 610-614.

(4) Lee, K.-H.; Sinnott, S. B. Nano Lett. 2005, 5, 793-798. 9870 .
(6) Wei, C.; Srivastava, D. Phys. Rev. Lett. 2003, 91, 235901.

(7) Skoulidas, A. I.; Ackerman, D. M.; Johnson, J. K.; Sholl, D. S. Phys. Rev. Lett. 2002, 89, 185901.

(8) Chen, H.; Johnson, J. K.; Sholl, D. S. J. Phys. Chem. B 2006, 110 , 1971-1975.

(9) Sokhan, V. P.; Nicholson, D.; Quirke, N. J. Chem. Phys. 2004, $120,3855-3863$.

(10) Jakobtorweihen, S.; Verbeek, M. G.; Lowe, C. P.; Keil, F. J.; Smit, B. Phys. Rev. Lett. 2005, 95, 044501.

(11) Jakobtorweihen, S.; Lowe, C. P.; Keil, F. J.; Smit, B. J. Chem. Phys. 2006, 124, 154706.

(12) Derouane, E. G.; André, J.-M. Chem. Phys. Lett. 1987, 137, 336340.

(13) Derouane, E. G.; André, J.-M.; Lucas, A. A. J. Catal. 1988, 110, $58-73$.

(14) Yashonath, S.; Santikary, P. J. Phys. Chem. 1994, 98, 6368-6376.

(15) Ghorai, P. K.; Yashonath, S.; Demontis, P.; Suffritti, G. B. J. Am Chem. Soc. 2003, 125, 7116-7123.

(16) Ghorai, P. K.; Yashonath, S. Chem. Phys. Lett. 2005, 402, $222-$ 226.

(17) Yashonath, S.; Rajappa, C. Faraday Discuss. 1997, 106, 105-118.

(18) Bhide, S.; Gosh, D.; Yashonath, S.; Ananthakrishna, G. Curr. Sci. 2004, 87, 971-975.

(19) Lowe, C. P. Europhys. Lett. 1999, 47, 145-151.

(20) Frenkel, D.; Smit, B. Understanding Molecular Simulations, From Algorithms to Applications; Academic Press: San Diego, 2002.

(21) Saito, R.; Fujita, M.; Dresselhaus, G.; Dresselhaus, M. S. Appl. Phys. Lett. 1992, 60, 2204-2206.

(22) Walther, J. H.; Jaffe, R.; Halicioglu, T.; Koumoutsakos, P. J. Phys. Chem. B 2001, 105, 9980-9987.

(23) Martin, M. G.; Siepmann, J. I. J. Phys. Chem. B 1998, 102, 25692577.

(24) Poling, B. E.; Prausnitz, J. M.; O'Connell, J. P. The Properties of Gases and Liquids, 5th ed.; McGraw-Hill: New York, 2000.

(25) Steele, W. A. The Interaction of Gaes with Solid Surfaces; Pergamon: Oxford, 1974.

(26) Yashonath, S.; Santikary, P. J. Phys. Chem. 1993, 97, 13 77813787. 